\title{
Lessons Learned From BaBar Silicon Vertex Tracker, Limits, and Future Perspectives of the Detector
}

V. Re, D. Kirkby, M. Bruinsma, S. Curry, J. Berryhill, S. Burke, D. Callahan, C. Campagnari, B. Dahmes, D. Hale, P. Hart, S. Kyre, S. Levy, O. Long, M. Mazur, J. Richman, J. Stoner, W. Verkerke, T. Beck, A. M. Eisner, J. Kroseberg, W. S. Lockman, G. Nesom, A. Seiden, P. Spradlin, W. Walkowiak, M. Wilson, C. Bozzi, G. Cibinetto, L. Piemontese, H. L. Snoek, D. Brown, E. Charles, S. Dardin, F. Goozen, L. T. Kerth, A. Gritsan, G. Lynch,

N. A. Roe, C. Chen, C. K. Lae, W. Hulsbergen, V. Lillard, D. Roberts, A. Lazzaro, F. Palombo, L. Ratti, P. F. Manfredi, E. Mandelli, C. Angelini, G. Batignani, S. Bettarini, M. Bondioli, F. Bosi, F. Bucci, G. Calderini, M. Carpinelli, M. Ceccanti, F. Forti, M. A. Giorgi, A. Lusiani, P. Mammini, G. Marchiori, M. Morganti, F. Morsani, N. Neri, E. Paoloni, A. Profeti, M. Rama, G. Rizzo, G. Simi, J. Walsh, P. Elmer, A. Perazzo, P. Burchat, A. Edwards, S. Majewski, B. A. Petersen, C. Roat, M. Bona, F. Bianchi, D. Gamba, P. Trapani, L. Bosisio, C. Cartaro, F. Cossuti, G. Della Ricca, S. Dittongo, S. Grancagnolo, L. Lanceri, L. Vitale, M. Datta, and A. Mihalyi

\begin{abstract}
The silicon vertex tracker (SVT) of the BaBar experiment at PEP-II is described. This is the crucial device for the measurement of the $B$ meson decay vertices to extract charge-conjugation parity (CP) asymmetries. It consists of five layers of double-
\end{abstract}

V. Re is with the Dipartimento di Fisica, Università di Bergamo, Bergamo, Italy and also with INFN-Pavia, I-24129 Bergamo, Italy.

D. Kirkby, M. Bruinsma, and S. Curry are with the University of California at Irvine, Irvine, CA 92697 USA.

J. Berryhill, S. Burke, D. Callahan, C. Campagnari, B. Dahmes, D. Hale, P. Hart, S. Kyre, S. Levy, O. Long, M, Mazur, J. Richman, J. Stoner, and W. Verkerke are with the University of California at Santa Barbara, Santa Barbara, CA 93106 USA.

T. Beck, A. M. Eisner, J. Kroseberg, W. S. Lockman, G. Nesom, A. Seiden, P. Spradlin, W. Walkowiak, and M. Wilson are with the Institute for Particle Physics, University of California at Santa Cruz, Santa Cruz, CA 95064 USA.

C. Bozzi, G. Cibinetto, and L. Piemontese are with the Dipartimento di Fisica, Università di Ferrara, Ferrara, Italy, and also with INFN-Ferrara, I-44100 Ferrara, Italy.

H. L. Snoek is with the National Institute for Nuclear Physics and High Energy Physics, NL-1009 DB Amsterdam, The Netherlands.

D. Brown, E. Charles, S. Dardin, F. Goozen, L. T. Kerth, A. Gritsan, G. Lynch, and N. A. Roe are with the Lawrence Berkeley National Laboratory, University of California, Berkeley, CA 94720 USA.

C. Chen, C. K. Lae, W. Hulsbergen, V. Lillard, and D. Roberts are with the University of Maryland, College Park, MD 20742 USA.

A. Lazzaro and F. Palombo are with the Dipartimento di Fisica, Università di Milano, Milano, Italy, and also with INFN-Milano, I-20133 Milano, Italy.

L. Ratti, P. F. Manfredi, and E. Mandelli are with the Dipartimento di Fisica, Università di Pavia, I-27100 Pavia, Italy.

C. Angelini, G. Batignani, S. Bettarini, M. Bondioli, F. Bosi, F. Bucci, G. Calderini, M. Carpinelli, M. Ceccanti, F. Forti, M. A. Giorgi, A. Lusiani, P. Mammini, G. Marchiori, M. Morganti, F. Morsani, N. Neri, E. Paoloni, A. Profeti, M. Rama, G. Rizzo, G. Simi, and J. Walsh are with the Dipartimento di Fisica, Scuola Normale Superiore Università di Pisa, Pisa, Italy and also with INFN-Pisa, I-56127 Pisa, Italy.

P. Elmer is with Princeton University, Princeton, NJ 08544 USA.

A. Perazzo is with the Stanford Linear Accelerator Center, Stanford, CA 94309 USA.

P. Burchat, A. Edwards, S. Majewski, B. A. Petersen, and C. Roat are with Stanford University, Stanford, CA 94305-4060 USA.

M. Bona, F. Bianchi, D. Gamba, and P. Trapani are with the Dipartimento di Fisica Sperimentale, Università di Torino, Torina, Italy and also with INFNTorino, I-10125 Torino, Italy.

L. Bosisio, C. Cartaro, F. Cossuti, G. D. Ricca, S. Dittongo, S. Grancagnolo, L. Lanceri, and L. Vitale are with the Dipartimento di Fisica, Università di Trieste, Trieste, Italy and also with INFN-Trieste, I-34127 Trieste, Italy.

M. Datta and A. Mihalyi are with the University of Wisconsin, Madison, WI 53706 USA. sided ac-coupled silicon strip detectors, read out by a full-custom integrated circuit, capable of simultaneous acquisition, digitization, and transmission of data. It represents the core of the BaBar tracking system, providing position measurements with a precision of $10 \mu \mathrm{m}$ (inner layers) and $30 \mu \mathrm{m}$ (outer layers). The relevant performances of the SVT are presented, and the experience acquired during the construction, installation, and the first five years of data-taking is described. Innovative solutions are highlighted, like the sophisticated alignment procedure, imposed by the design of the silicon tracker, integrated in the beamline elements and mechanically separated from the other parts of BaBar. The harshness of the background conditions in the interaction region required several studies on the radiation damage of the sensors and the front-end chips, whose results are presented. Over the next five years the luminosity is predicted to increase by a factor three, leading to radiation and occupancy levels significantly exceeding the detector design. Extrapolation of future radiation doses and occupancies is shown together with the expected detector performance and lifetime. Upgrade scenarios to deal with the increased luminosity and backgrounds are discussed.

\section{Index Terms-Radiation damage, silicon detector.}

\section{INTRODUCTION}

$\mathbf{T}$ HE BaBar experiment [1] at the PEP-II $e^{+} e^{-}$storage ring is designed to primarily measure charge-conjugation parity $(\mathrm{CP})$ violation in the $B \bar{B}$ system and to over-constrain the quark-mixing matrix. Many of these measurements require a precise measurement of the difference between the decay times of the two $B$ mesons. The $B \bar{B}$ pairs are produced in $\Upsilon(4 S)$ meson decays and in order to separate the two $B$ decays, the $\Upsilon(4 S)$ meson is given a boost of $\beta \gamma=0.56$ in the lab frame by having asymmetric beam energies. This gives an average $280 \mu \mathrm{m}$ separation of the $B$ decay vertices.

Precision measurements of $\mathrm{CP}$ violation require large amounts of $B$ decays and PEP-II has therefore been designed to deliver high luminosities $\left(10^{33}-10^{34} \mathrm{~cm}^{-2} \mathrm{~s}^{-1}\right)$. The high luminosities are associated with relatively high radiation doses and the BaBar silicon vertex tracker (SVT) is therefore designed to not only provide excellent vertex resolution, but also be radiation hard. However, both the luminosity and radiation doses have exceeded design and are projected to continue to 


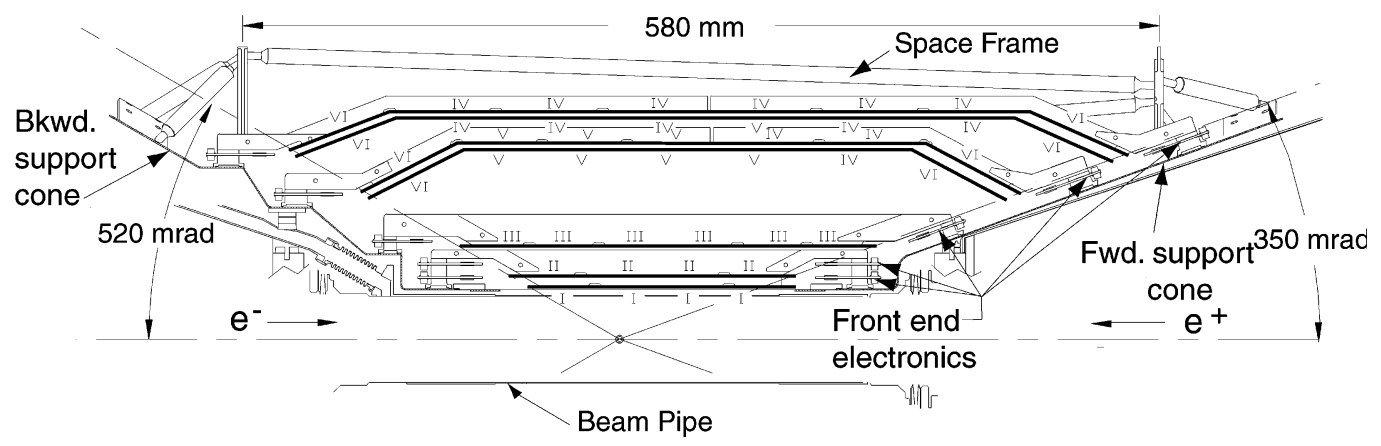

Fig. 1. Longitudinal cross section of the SVT.

rise over the next five years. Considerable effort have gone into understanding the future performance and radiation limitations of the existing system and whether any replacement will be needed. We will report on these studies here.

\section{THE BABAR SILICON VERTEX TRACKER}

The SVT has two tasks. First, $B$ and $D$ decay vertices have to be accurately reconstructed in order to measure the difference in decay length, $\Delta z$, between the two $B$ mesons. With the design $\Delta z$ resolution of $130 \mu \mathrm{m}$, the precision of a $\mathrm{CP}$ violation measurement is only $10 \%$ worse than with a perfect $\Delta z$ measurement. Achieving this $\Delta z$ resolution requires a single vertex resolution better than $80 \mu \mathrm{m}$. Second, low transverse-momentum $\left(p_{T}<120 \mathrm{MeV} / \mathrm{c}\right)$ particles, such as pions from $D^{*}$ decays, do not fully traverse the main BaBar tracking chamber and therefore have to be reconstructed in the SVT alone.

The SVT design [2], [3] accomplishes these goals by having five layers of double-sided, ac-coupled silicon micro-strip detectors in a concentric configuration around the beam pipe as shown in Fig. 1. Each layer is built from 6 to 18 modules, where each module itself consists of several $300 \mu \mathrm{m}$ thick silicon detectors glued together. The modules are mechanically supported by Kevlar/carbon-fiber along the modules, while the detector signals are carried by flexible fanout circuits to the front-end electronics located at both ends of each module. The readout of a single module is divided into four so-called readout sections. The strips on the two sides of a modules are perpendicular and provide a $z$ and $r$ - $\phi$ measurement. The three inner layers are planar, located very close to the interaction point and have a readout pitch of $50-110 \mu \mathrm{m}$. This provides the resolution in track angle and impact parameter measurements needed to get a precise vertex determination. The two outer layers are of most importance to the track finding, particularly for low momentum particles. The silicon detectors at the end of layer 4 and 5 modules are at an angle to the beam pipe in order to provide the largest possible angular acceptance, while reducing the amount of material traversed by low-angle tracks. The polar angle acceptance is $20^{\circ}<\theta<150^{\circ}$ and is mainly constrained by the design of the PEP-II interaction region.

More than 150000 silicon strips are read out using a fully custom-designed chip, known as AToM [4], located at both ends and sides of each module. Each AToM chip is capable of simultaneous acquisition, digitization, and sparsified readout of
128 channels. The linear analog section consists of a charge sensitive preamplifier, followed by a shaper. The output signal is compared to a programmable threshold producing a logic pulse whose width [time over threshold (ToT)] is approximately proportional to the logarithm of the collected charge. The logic pulse is digitized at $15 \mathrm{MHz}$ and stored into a latency buffer. Upon a level-1 trigger signal, a $1 \mu \mathrm{s}$ window in the buffer is searched for the first hit. For channels with a hit, the ToT and hit time in the buffer is transmitted to the data acquisition system.

\section{Performance And Alignment}

Of the 208 readout sections in the SVT, nine failed during or right after their installation in 1999. During a shutdown in 2002 , four of the failures were identified as slipped connectors and fixed. No readout section has failed during running due to radiation damage or otherwise.

The hit efficiency is measured by counting hits on tracks passing through the active area of the silicon sensors. Excluding the five broken readout sections, the average hit efficiency is 97\%, which includes inefficiencies due to broken capacitors and dead channels in the AToM chips. The SVT hit resolution is measured using high momentum tracks in two-prong events. For tracks with a $90^{\circ}$ incidence angle to the silicon modules, the hit resolution is $10-15 \mu \mathrm{m}$ in the inner three layers and 30-40 $\mu \mathrm{m}$ for the outer two layers. With this, the SVT achieves its design of an average vertex resolution of better than $80 \mu \mathrm{m}$. The $\Delta z$ resolution is $190 \mu \mathrm{m}$, dominated by uncertainties in inclusively reconstructing one of the two $B$ vertices.

Tracking efficiencies for low momentum particles, where the SVT contribution is essential, have been studied in data using slow pions from $D^{* \pm}$ decays. These studies give an tracking efficiency above $75 \%$ for momentum above $100 \mathrm{MeV} / \mathrm{c}$ and above $90 \%$ for momentum above $200 \mathrm{MeV} / \mathrm{c}$.

The deposited charge measured by the ToT mechanism gives a reasonable ionization loss measurement, $d \mathrm{E} / d x$, when averaged over hits in all five layers. Using a $60 \%$ truncated mean, the $d \mathrm{E} / d x$ resolution on minimum ionizing particles is approximately $14 \%$. For low momentum tracks this can be used for particle identification. We achieve better than $2 \sigma$ separation between kaons and pions with momentum below $500 \mathrm{MeV} / \mathrm{c}$ and between kaons and protons below $1 \mathrm{GeV} / \mathrm{c}$.

To achieve the SVT performance given above, the SVT needs to be carefully aligned. In BaBar this involves two separate procedures. First, the internal alignment of the SVT and second, the 
global alignment of the SVT with respect to the rest of the detector. The internal alignment determines the location and orientation of the 340 individual silicon wafers in the SVT. Furthermore, it has been found that the inner layers are not perfectly planar and an additional parameter is used to describe the bending of the wafers in those layers. To determine the alignment parameters, tracks from several classes of events are used: dimuon event, cosmic events, high- $p_{T}$ tracks, and tracks through overlapping SVT modules. The alignment procedure minimizes the $\chi^{2}$ calculated from hit residuals and track fit errors. A further constraint is the results of an optical survey of the individual modules performed during the assembly of the SVT. The internal alignment is typically updated every $1-2$ months. The global alignment treats the SVT as a rigid body and aligns it with respect to the main tracking chamber. This procedure is much simpler, but needs to be done every hour as the SVT have diurnal movements.

\section{RADiATION DAMAgE STUdies}

The performance of the PEP-II storage ring has been excellent, delivering more than $250 \mathrm{fb}^{-1}$ by the summer of 2004 and achieving an instantaneous luminosity in excess of $9 \times 10^{33} \mathrm{~cm}^{-2} \mathrm{~s}^{-1}$, three times the design value. However, the radiation backgrounds have also been higher than foreseen. The total radiation dose over the full lifetime of BaBar was not expected to exceed $2 \mathrm{Mrad}$, but certain parts of the detector have already received a higher dose. The inner layers naturally absorb the most dose and due to the design of the interaction region, the radiation is concentrated in a $1-\mathrm{cm}$ wide band in the horizontal. Away from the horizontal band, the radiation levels are up to a factor 10 lower at the same radius.

Given that BaBar is expected to continue data-taking until 2009 with ever increasing luminosities, and thus radiation doses, the SVT group has performed extensive radiation tests to determine the lifetime of the SVT. Of particular concern was the possibility of a partial replacement of damaged modules in 2005. Both the silicon modules themselves and the front-end electronics were tested for radiation hardness. The results of this are reported below.

\section{A. Silicon Module Studies}

PEP-II is an $e^{+} e^{-}$collider and the vast majority of the radiation dose in the SVT is therefore electromagnetic in nature. Consequently all SVT radiation studies have been performed with either electron beams or gamma rays. For the silicon modules, the concern is bulk damage and the irradiations [5] have therefore been performed with $0.9 \mathrm{GeV}$ and $3 \mathrm{GeV}$ electron beams at Elettra (Trieste) and SLAC, respectively. Irradiation was done in several steps up to a total dose of $10 \mathrm{Mrad}$. The bulk damage is observed as an increased leakage current, changes in the depletion voltage and decreased charge collection efficiency. The increase in leakage current causes an increase in noise which scales as the square root of the channel current. During radiation tests a linear increase of $2 \mu \mathrm{A} / \mathrm{cm}^{2} / \mathrm{Mrad}$ at $23{ }^{\circ} \mathrm{C}$ was observed. This is consistent with increases measured in the installed modules.
The depletion voltage was measured by measuring interstrip capacitance and charge collection efficiency as a function of the applied bias voltage. Initially the depletion voltage dropped until a dose of $3 \pm 1 \mathrm{Mrad}$ was reached at which point type-inversion occurred and the depletion voltage started to increase again. The electrical properties of the modules (edge currents, interstrip capacitance, and insulation between the p-strips) did not change significantly after the type-inversion, showing that the detector can still be operated after that point. Even after $10 \mathrm{Mrad}$, the depletion voltage is below $70 \mathrm{~V}$, giving us confidence that we will continue to be able to fully deplete the heavily irradiated modules.

The charge collection efficiency was determined by illuminating parts of a module with a light emitting diode at a penetrating wavelength $(\lambda=1060 \mathrm{~nm})$. The detector response as a function of the light intensity was measured before and after irradiation, giving a precise measurement of the change in charge collection efficiency for each readout channel. The average drop in charge collection efficiency was $1 \% / \mathrm{Mrad}$.

\section{B. Front-End Electronics}

A set of AToM chips connected in a half-module, similar to installed ones, were irradiated with $1 \mathrm{MeV} \gamma$-rays from ${ }^{60} \mathrm{Co}$ sources at SLAC and LBL. The chips were powered and supplied with an external clock signal during irradiation and continued to work throughout the irradiation. The irradiation continued up until $5 \mathrm{Mrad}$. The electronics noise was observed to increase by $15-20 \% / \mathrm{Mrad}$, while the gain decreased by about $4 \% /$ Mrad. Noise and gain measurements for the installed SVT modules over the last five years show comparable changes in gain and noise levels. Recently, AToM chips have been irradiated with a narrow electron beam up to $10 \mathrm{Mrad}$ in an effort to further understand the pedestal change described in the next section. Again comparable noise and gain changes were measured with no digital failures.

\section{Unforeseen Effects}

Despite the extensive tests several unexpected radiation related effects were observed in the installed detector. Fortunately the SVT was designed with sufficient flexibility that we could work around the effects with essentially no loss in detector efficiency.

After an accumulated dose of about 1 Mrad, we measured an increase in the pedestal of the signal into the comparator in the AToM, requiring an increased threshold level in order to keep the noise occupancy at a reasonable low level. The increase was first observed in the readout channels exposed to the highest amount of radiation, but other channels followed as soon as their accumulated dose reached about $1 \mathrm{Mrad}$. This is illustrated in Fig. 2, which shows the measured pedestal versus the radiation dose in the most irradiated channels. The readout channels in the AToM chip are geometrically offset in azimuth with respect to the silicon strips, showing that it is the most irradiated readout channel, not silicon strip which changes first. From this we conclude that the radiation damage is in the AToM chip itself. The pedestal continues to rise until an additional $400 \mathrm{krad}$ of dose has been accumulated, after which it starts to fall back toward 


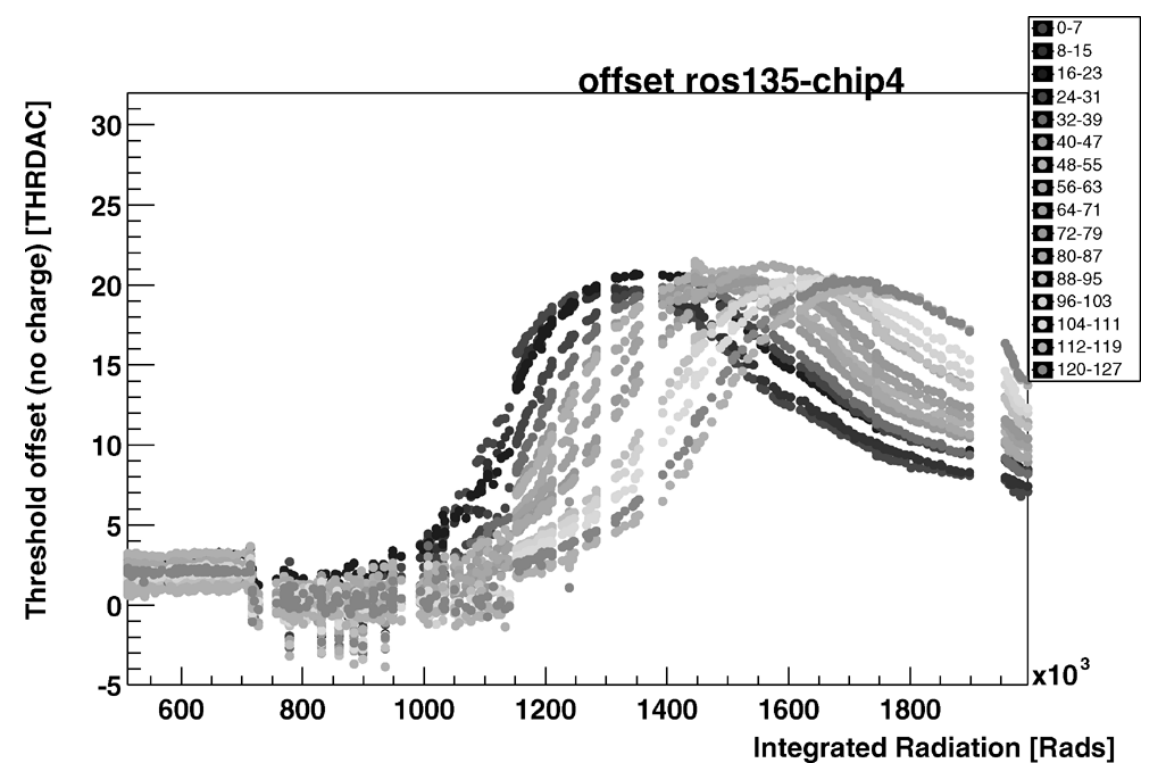

Fig. 2. Pedestals averaged over groups of eight readout channels versus radiation dose averaged over the most irradiated channels. One THRDAC count corresponds to a charge of $0.05 \mathrm{fC}$.

its original value. The pedestals peak at roughly half the dynamic range of the threshold DAC, corresponding to roughly $1 \mathrm{fC}$. This effect is qualitatively understood as an imbalance in the comparator circuit caused by the nonuniform irradiation. After sufficient irradiation the balance slowly gets restored. The effect did not show up in the initial irradiation tests with ${ }^{60} \mathrm{Co}$ as that had a uniform radiation field. Subsequent radiation tests [6] with a narrow electron beam reproduced the pedestal shift. Operationally the noise is kept under control by increasing the threshold used in the readout. However, this can only be set on a chip-by-chip basis leading to an inefficiency for small charges in channels, where the pedestal has not yet increased. A procedure has been devised which selects the optimal threshold for each chip balancing the inefficiencies from noise occupancy and minimum charge requirement. The thresholds need to be reoptimized about every two months.

A second effect has occurred more recently. Starting in March 2004 , the leakage current in several layer four modules suddenly started to increase from a few $\mu \mathrm{A}$ to hundreds of $\mu \mathrm{A}$ over a period of three months. At such a high current level, the occupancy is increased significantly due to noise, resulting in a hit efficiency loss. In the same period no significant leakage increase was observed in any of the inner layers, which receive higher radiation levels, and thus it could not be caused by radiation damage to the silicon bulk. However, the leakage current would only increase during time periods with both radiation and a fully biased detector. If either of the two conditions were not satisfied, the leakage current would drop at roughly the same rate it had been increasing. Eventually the rate of increase in leakage current was found to depend on the voltage difference between layer four and five. Normally the outer p-side of a layer four module is at $-20 \mathrm{~V}$, while the inner $\mathrm{n}$-side of a layer five module is at $+20 \mathrm{~V}$ giving a $+40 \mathrm{~V}$ voltage gap. Reducing the voltage gap to $0 \mathrm{~V}$ caused the leakage current to drop again with no detrimental effect on the SVT performance.
This curious effect is believed to be caused by static charge accumulation. Ions are produced in the gap between layer four and five by radiation and accumulates on the $\mathrm{SiO}_{2}$ passivation layer on the p-side of layer four. The accumulated charge increases the field at the edge of the $\mathrm{p}^{+}$-implant junction, eventually causing junction breakdown with increased leakage current as a result. The effect of accumulated charge has been qualitatively understood in simulation and in lab tests with ionized air. Only layer four is affected due to its particular configuration of $\mathrm{p}$ - and $\mathrm{n}$-sides with respect to the other layers. The effect was not observed before, because in the past SVT was normally unbiased for about 10 min every hour in order to refill the storage ring. Now PEP-II has continuous injection and the SVT can stay biased for many hours at a time and thus not allowing accumulated charge to dissipate. By keeping the voltage gap low, we have successfully remedied the high leakage currents, which are now all below $50 \mu \mathrm{A}$, and are not expected to cause problems in the future.

\section{Expected Performance}

Based on the radiation studies described above, the expected noise levels as a function of radiation dose is shown in Fig. 3. The degradation of the front-end electronics is seen to dominate. In the same figure the signal-to-noise ratio $(S / N)$ is shown taking into account the loss in signal from the charge-collection efficiency and gain losses. At 5-6 Mrad of dose, a $S / N$ of 10 is reached, at which point studies shows the detector performance starts to be seriously impacted. Modules are still expected to function after even higher doses, but the 5-6 Mrad is a reasonable measure of the expected lifetime of SVT modules.

\section{RADIATION AND OCCUPANCY PREDICTIONS}

The radiation dose accumulated by the SVT is measured by 12 silicon PIN-diodes located next to the AToM chips of the 


\section{SVT L1-Signal/Noise vs dose}

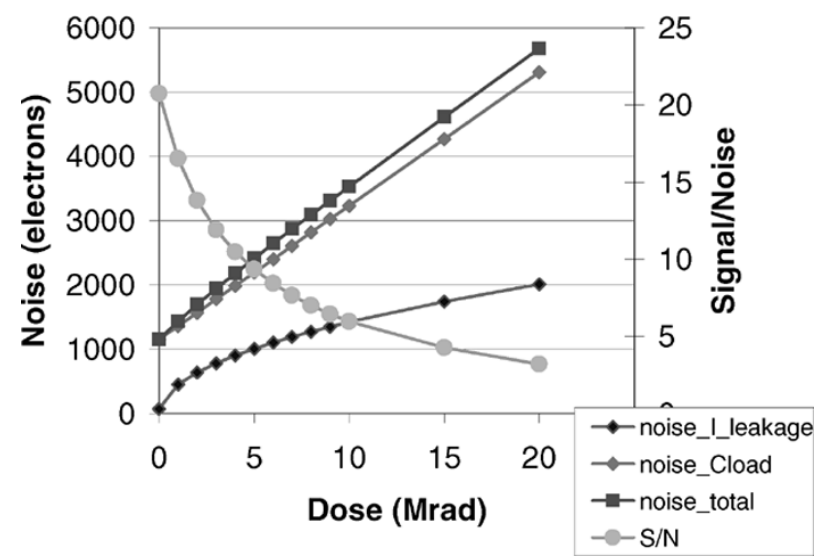

Fig. 3. Individual noise components and total predicted noise versus radiation dose. Also shown is the signal-to-noise ratio taking into account efficiency losses.

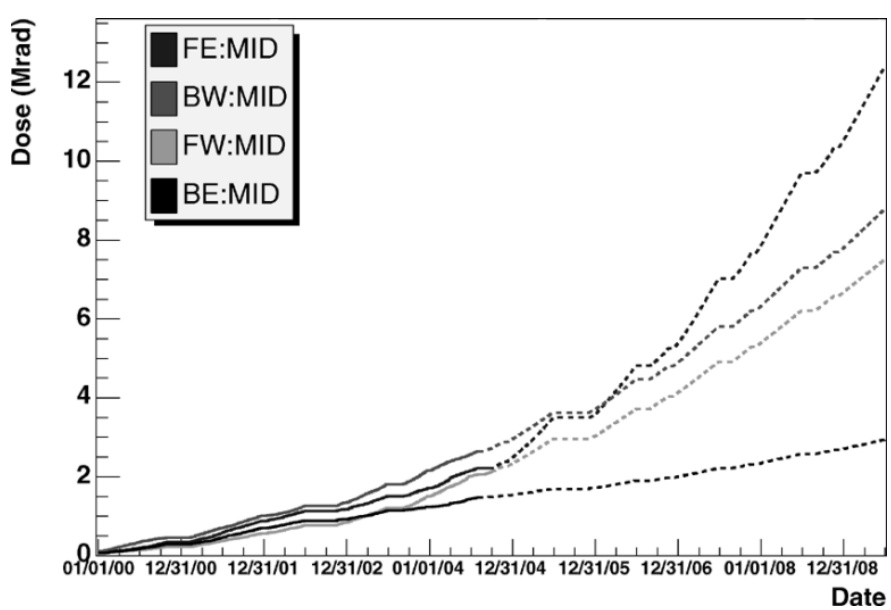

Fig. 4. Measured and predicted radiation doses in four locations in the horizontal plane as a function time. The solid lines are measured doses, while the dashed lines are predictions based on predicted beam currents and luminosity.

innermost layer. Fig. 4 shows the evolution in dose as measured by the four PIN-diodes located in the horizontal plane. Also shown is the doses predicted until 2009. The dose prediction is based on measured dose rates parameterized as a function of beam currents and luminosity together with the predicted future beam currents and luminosity. The beam currents are predicted to increase by $50 \%-100 \%$, while the luminosity should go up by a factor three. A significant uncertainty is associated both with the predicted beam currents/luminosity and with the dose rate dependence. By 2006, the first modules are predicted to reach a dose of $5 \mathrm{Mrad}$, where performance starts to degrade, and by 2009 some areas will have seen more than twice that dose. However, the dose away from the horizontal plane is much lower than 5 Mrad, even by 2009, and in the end only 5\%-10\% of the readout channels in the two inner layers are expected to be significantly affected by radiation damage.

Radiation damage is not the only thing impacting the SVT performance. With increased radiation levels, the hit occupancy from beam backgrounds also increase and can reach a level where the performance is negatively impacted. Background hits can shadow hits from particles coming from physics processes, distort the measured charge, and degrade the hit resolution. At very high occupancies the track finding is also affected. The effect of high occupancy has been studied using data recorded under bad background conditions typically caused by high pressure in the beam vacuum system. These studies show a linear decrease in hit efficiency and hit resolution as a function of hit occupancy. At $20 \%$ hit occupancy, the hit efficiency is observed to decrease by about $10 \%$, while the hit resolution in the inner layers more than double to about $25 \mu \mathrm{m}$. The $20 \%$ occupancy is therefore used as a rough limit on tolerable occupancy. Note that the $20 \%$ background occupancy is reduced by roughly a factor three before the tracking algorithms, by using the time information of the hits.

Similar to the accumulated dose, the future occupancy can be predicted by parameterizing the occupancy as a function of beam currents and luminosity and extrapolating to expected future beam currents and luminosity. The SVT already has a few local spots with background occupancy higher than $20 \%$ and by 2009 we expect about $10 \%$ of the two innermost layers to be above this limit. There is a high overlap between radiation damaged readout channels and high occupancy channels. However, it is not $100 \%$ identical due to a geometrical offset of the readout chips with respect to the strips and due to many high occupancy $z$-strips. The high-degree overlap means that it makes little sense to replace the radiation damaged modules with new, identical modules as they will anyway be seriously degraded in performance by high background occupancy.

The impact of a significant degradation in $10 \%$ of the inner two layers have been studied in simulation by disabling varying parts of the modules in the horizontal plane. In many physics channels, such as $B^{0} \rightarrow J / \psi K_{S}^{0}$, the reconstruction efficiency drops by just a few percent, while the invariant mass and time resolution is essentially unaffected. The most significant loss observed is in the reconstruction of slow pions from $D^{*}$ decays, which drops by about $10 \%$ when $10 \%$ of the inner two layers are turned off. These performance losses are judged to be acceptable and the SVT is planned to remain unchanged until 2009.

\section{CONCLUSION}

The BaBar SVT has had excellent performance during the last five years and has been an essential part of the BaBar experiment. A few unforeseen problems have arisen, but due to the flexibility of the system, they have been remedied without loss in performance. Over the next five years the luminosity is expected to increase by a factor three, while the radiation dose will increase even more. Extensive radiation studies have therefore been carried out, resulting in an expected SVT module lifetime of about 5-6 Mrad. This is significantly better than the original design requirement of 2 Mrad. Extrapolating the accumulated dose to $2009,5 \%-10 \%$ of the inner-layer readout channels are expected to exceed the new radiation budget. However, most of the high radiation areas will also be performance limited by high occupancy. Therefore, it has been decided not to exchange any radiation damaged modules. The physics impact from this has been evaluated to be negligible. 


\section{REFERENCES}

[1] B. Aubert et al., "The BABAR detector," Nucl. Instrum. Methods Phys. Res. A, vol. A479, pp. 1-116, 2002.

[2] “The BaBar Technical Design Report,” SLAC-R-95-457, Menlo Park, CA, 1995.

[3] D. Barbieri et al., "Silicon sensors for the BABAR vertex tracker: Design, electrical tests and production quality control," Nuove Cimento A, vol. A112, pp. 113-130, 1999.
[4] V. Re et al., "The rad-hard readout system of the $B A B A R$ silicon vertex tracker," Nucl. Instrum. Methods Phys. Res. A, vol. A409, pp. 354-359, 1998.

[5] V. Re et al., "Performance, radiation damage and future plans of the BABAR silicon vertex tracker," in IEEE Trans. Nuclear Sci., vol. 51, Oct. 2004, pp. 2298-2301.

[6] S. Bettarini et al., "Study of the radiation hardness of irradiated AToM front-end chips of the BaBar silicon vertex tracker," in Proc. $2004 \mathrm{Nu}$ clear Science Symp., Rome, Italy, 2004. 